\title{
Atrial flow regulator as a novel therapy for patients with chronic heart failure
}

\author{
Łukasz Lewicki ${ }^{1,2 *}$, Robert Sabiniewicz ${ }^{3 *}$, Janusz Siebert ${ }^{1,4}$, Marek Szołkiewicz ${ }^{2}$ \\ ${ }^{1}$ University Center for Cardiology, Gdansk, Poland \\ ${ }^{2}$ Department of Cardiology and Angiology, Kashubian Center for Heart and Vascular Diseases, \\ Pomeranian Hospitals, Wejherowo, Poland \\ ${ }^{3}$ Department of Pediatric Cardiology and Congenital Heart Disease, Medical University of Gdansk, Poland \\ ${ }^{4}$ Department of Family Medicine, Medical University of Gdansk, Poland
}

Mortality and morbidity among patients with heart failure (HF) remain high, despite advances in therapy.

Heart failure with preserved ejection fraction $(\mathrm{HFpEF})$ is driven by diminished left ventricle relaxation and elevated filling pressures, all of which lead to pulmonary congestion [1,2]. In these patients, therapeutic options impacting prognosis are limited.

Recently, a novel therapy has been proposed. It is based on creating a communication between both atria using a trans-septal puncture and balloon septostomy. The concept is based on the wellknown Lutembacher syndrome, which is defined as a combination of mitral stenosis and atrial septal defect (ASD). The left to right interatrial shunt enables decompression of the left atrium and thus, may improve patient symptoms.

Moreover, observations made in elderly subjects with masked left ventricle restriction, who underwent ASD closure, showed that temporary ASD occlusion with a balloon resulted in significant elevated left atrial pressure [3]. A fenestrated ASD septal occlude has been designed to enable bidirectional flow both in systolic and diastolic impairment [4]. Implantable pressure systems have provided data that left atrial pressure is highly variable over the course of a day and sustained elevations precede clinical events, averaging $>25 \mathrm{mmHg}$ for several days before admission or death [5].

Therefore, a therapy focused on decreasing left atrial filling pressures seems to be promising.
Several small studies proved initial safety and efficacy of three different interatrial shunting devices in therapy for patients either with heart failure with reduced ejection fraction ( $\mathrm{HFrEF}$ ) or HFpEF [6, 7]. There are three different devices available for patients with either HFrEF or HFpEF: interatrial shunt device (IASD, Corvia Medical Inc., Tewksbury, MA, USA), V-Wave shunt (V-Wave Ltd., Caesarea, Israel) and Atrial Flow Regulator (AFR, Occlutech, Heslingborg, Sweden).

Atrial Flow Regulator is a self-expandable a double-disc nitinol wire mesh construction allowing communication across the interatrial septum (Fig. 1). In contrast to V-wave and IASD, it is available in different sizes. The offered fenestration diameter ranges from 4 to $10 \mathrm{~mm}$, but for $\mathrm{HF}$ patients only $8 \mathrm{~mm}$ and $10 \mathrm{~mm}$ have the European Conformité Européenne (CE) mark. Additionally, there are two available heights of the device: 5 and $10 \mathrm{~mm}$, chosen according to interatrial septal thickness. The device is repositionable and retrievable.

First AFR implantation in Poland was done in a 28-year-old patient with severe pulmonary arterial hypertension (PAH) [8]. More recently, AFR has been successfully used as a bridge to lung transplantation in a young patient with drugresistant idiopathic PAH [9].

Recently four AFR devices in patients with severe HFrEF were successfully implanted. These procedures were done as a part of ongoing PROLONGER trial (Pomeranian atRial flOw

Address for correspondence: Łukasz Lewicki, MD, PhD, University Center for Cardiology, ul. Dębinki 2, 80-211 Gdańsk, Poland, tel: +48 501702 885, e-mail: luklewicki@gmail.com

Received: 8.03.2020 Accepted: 16.05 .2020

*The first two authors contributed equally to this work. 


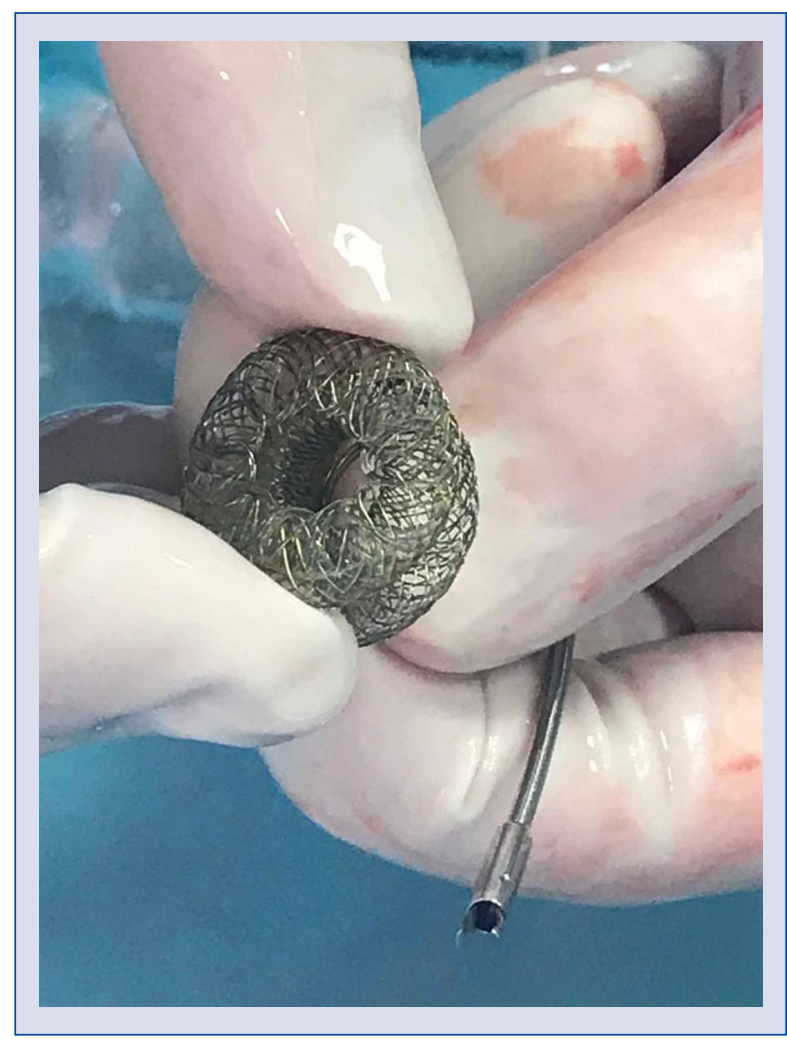

Figure 1. Atrial Flow Regulator (AFR, Occlutech, Heslingborg, Sweden).

reguLatOr iN conGestive hEart failuRe; No. NCT04334694 at clinicaltrials.gov).

Herein presented, are the results of a 66 -year-old male with HFrEF (LVEF 25\%), history of three myocardial infarctions, arterial hypertension and paroxysmal atrial fibrillation. Despite an optimal therapy, the patient remained symptomatic with New York Heart Association (NYHA) III. His 6 -minute walk test distance (6MWT) was $200 \mathrm{~m}$. The patient underwent diagnostic right heart catheterization with the Swan-Ganz catheter, which revealed decreased cardiac output accompanied with significantly increased pulmonary artery wedge pressure (PAWP).

According to PROLONGER protocol, hemodynamic indications for AFR are: PAWP above $15 \mathrm{mmHg}$ or $25 \mathrm{mmHg}$ at rest and exertion respectively. A right atrial pressure above $20 \mathrm{mmHg}$ or exceeding PAWP is the contraindication for an atrial shunting procedure.

The AFR procedure was performed under general anesthesia. A three-dimensional transesophageal echocardiography (TEE) guided trans-septal puncture was performed followed by a $12 \mathrm{~mm}$ balloon septostomy. An AFR (8 mm fenestration, $5 \mathrm{~mm}$ height) was successfully implanted using

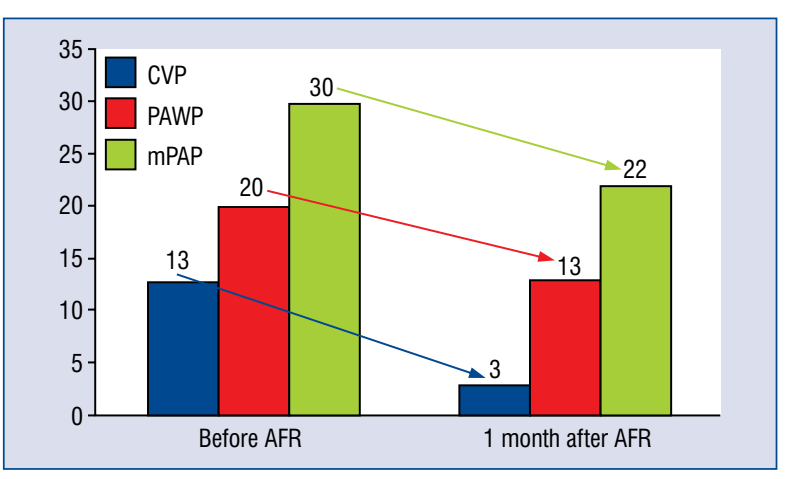

Figure 2. Hemodynamic parameters taken from diagnostic right heart catheterization before, and 1 month after implantation of Atrial Flow Regulator (AFR); CVP - central venous pressure; PAWP — pulmonary artery wedge pressure; mPAP — mean pulmonary artery pressure.

a $12 \mathrm{~F}$ dedicated delivery system. The left to right mean gradient obtained from TEE was $2.7 \mathrm{mmHg}$. The patient was discharged home on the third day. As the patient had a history of atrial fibrillation, he was given non-vitamin $\mathrm{K}$ antagonist oral anticoagulants. There was no other specific indication for anticoagulation in this patient, because flow through the device was left to right.

The first follow-up visit after 1 month was complete, significant clinical improvement was noticed. The patient moved from NYHA III to NYHA II and 6MWT distance increased from $200 \mathrm{~m}$ to $397 \mathrm{~m}$. Diagnostic right heart catheterization revealed significant reduction in PAWP, mean pulmonary artery and right atrial pressures (Fig. 2). The mean left to right gradient in TEE was $10 \mathrm{mmHg}$ compared to $2.7 \mathrm{mmHg}$ directly after AFR implantation. This difference could be explained by the fact that left atrial pressure had changed dynamically according to fluid overload, exertion and other conditions. Similar variability in all 4 patients thus far were observed.

The second follow-up visit was scheduled at 2 months.

This experience with AFR device is a promising option for patients with severe $\mathrm{HF}$ and further results will be published soon.

\section{Conflict of interest: None declared}

\section{References}

1. Lam CSP, Voors AA, de Boer RA, et al. Heart failure with preserved ejection fraction: from mechanisms to therapies. Eur Heart J. 2018; 39(30): 2780-2792, doi: 10.1093/eurheartj/ehy301, indexed in Pubmed: 29905796. 
2. Borlaug BA, Paulus WJ. Heart failure with preserved ejection fraction: pathophysiology, diagnosis, and treatment. Eur Heart J. 2011; 32(6): 670-679, doi: 10.1093/eurheartj/ehq426, indexed in Pubmed: 21138935.

3. Ewert P, Berger F, Nagdyman N, et al. Masked left ventricular restriction in elderly patients with atrial septal defects: a contraindication for closure? Catheter Cardiovasc Interv. 2001; 52(2): 177-180, doi: 10.1002/1522-726x(200102)52:2<177::aidccd1043>3.0.co;2-g, indexed in Pubmed: 11170324.

4. Bruch L, Winkelmann A, Sonntag S, et al. Fenestrated occluders for treatment of ASD in elderly patients with pulmonary hypertension and/or right heart failure. J Interv Cardiol. 2008; 21(1): 44-49, doi: 10.1111/j.1540-8183.2007.00324.x, indexed in Pubmed: 18086135.

5. Ritzema J, Troughton R, Melton I, et al. Physician-directed patient self-management of left atrial pressure in advanced chronic heart failure. Circulation. 2010; 121(9): 1086-1095, doi: 10.1161/CIRCULATIONAHA.108.800490, indexed in Pubmed: 20176990.

6. Shah SJ, Feldman T, Ricciardi MJ, et al. One-Year safety and clinical outcomes of a transcatheter interatrial shunt device for the treatment of heart failure with preserved ejection fraction in the reduce elevated left atrial pressure in patients with heart failure (REDUCE LAP-HF I) trial: a randomized clinical trial. JAMA Cardiol. 2018; 3(10): 968-977, doi: 10.1001/jamacardio.2018.2936, indexed in Pubmed: 30167646.

7. Paitazoglou C, Özdemir R, Pfister R, et al. The AFR-PRELIEVE trial: a prospective, non-randomised, pilot study to assess the Atrial Flow Regulator (AFR) in heart failure patients with either preserved or reduced ejection fraction. EuroIntervention. 2019; 15(5): 403-410, doi: 10.4244/EIJ-D-19-00342, indexed in Pubmed: 31130524.

8. Dąbrowska-Kugacka A, Ciećwierz D, Żuk G, et al. Atrial flow regulator for severe drug resistant pulmonary arterial hypertension after congenital heart defect correction. Cardiol J. 2019; 26(1): 102-104, doi: 10.5603/CJ.2019.0016, indexed in Pubmed: 30882193.

9. Janus M, Sławek-Szmyt S, Araszkiewicz A, et al. Atrial flow regulator as a bridge to lung transplantation in a young patient with drug-resistant idiopathic pulmonary arterial hypertension. Kardiol Pol. 2020; 78(5): 461-462, doi: 10.33963/KP.15178, indexed in Pubmed: 32024808. 